\title{
23. GEOCHEMISTRY OF CARBON: DEEP SEA DRILLING PROJECT LEG 44
}

\author{
J.G. Erdman and K.S. Schorno, Phillips Petroleum Company, Bartlesville, Oklahoma
}

\section{INTRODUCTION}

Thirty-seven frozen core sections were obtained through the JOIDES Organic Geochemistry Panel for organic geochemical study. These samples consisted of Pleistocene through Jurassic (upper Tithonian) sections from Holes 388A, 390, 390A, and 391A, 391B, 391C from the lower continental rise hills, the Blake Nose, and Blake-Bahama Basin on the outer North Atlantic continental margin. Sample site locations are shown in Figure 1.

\section{SAMPLING AND STUDY PROCEDURES}

The flat faces of the frozen half-sections were smoothed by milling upon arrival in our laboratory. Because of space limitations, black and white photographs for only those samples that have been extensively disturbed and mixed as a consequence of drilling, namely, Sections 390-3-3, 390A-6-0, 391C-9-3, and 391C-12-4 are shown in Figure 2. Each section was then described and chips of each textural type removed to determine the content of organic and carbonate carbon. These values are listed in Table 1.

The remainder of the core section was freed as thoroughly as possible of contaminating mud and characterized as shown in the flow sheet in Figure 3. The sample was freeze dried, crushed, and sieved to 150 $\mu \mathrm{m}$. A 2-gram portion of homogenized core was analyzed for organic and carbonate carbon and for ammonium and organic nitrogen. The remaining freeze-dried material was extracted by treatment with methylene chloride for 24 hours. The methylene chloride was removed yielding an oil extract which hereafter is designated as the lipid fraction. When sufficient sample was available, elemental analyses were performed for lipid carbon, hydrogen, nitrogen, and sulfur.

The lipid fraction was chromatographed over activated silica gel using as eluants, pentane, methylene chloride, and a 50:50 mixture of methylene chloride methanol to obtain "saturate," "aromatic," and "asphaltic" fractions. The $n$-alkane portion of the saturate fraction was adducted in urea and analyzed by gas chromatography.

Carbon isotopic compositions were determined by closed loop combustion on an AVCO isotope ratio mass spectrometer. Measurements were made on the lipid and its aromatic and asphaltic subfractions, where possible, and on the kerogen fraction.

\section{RESULTS}

Average numerical data generated for the samples along with sub-bottom depth and age are provided in

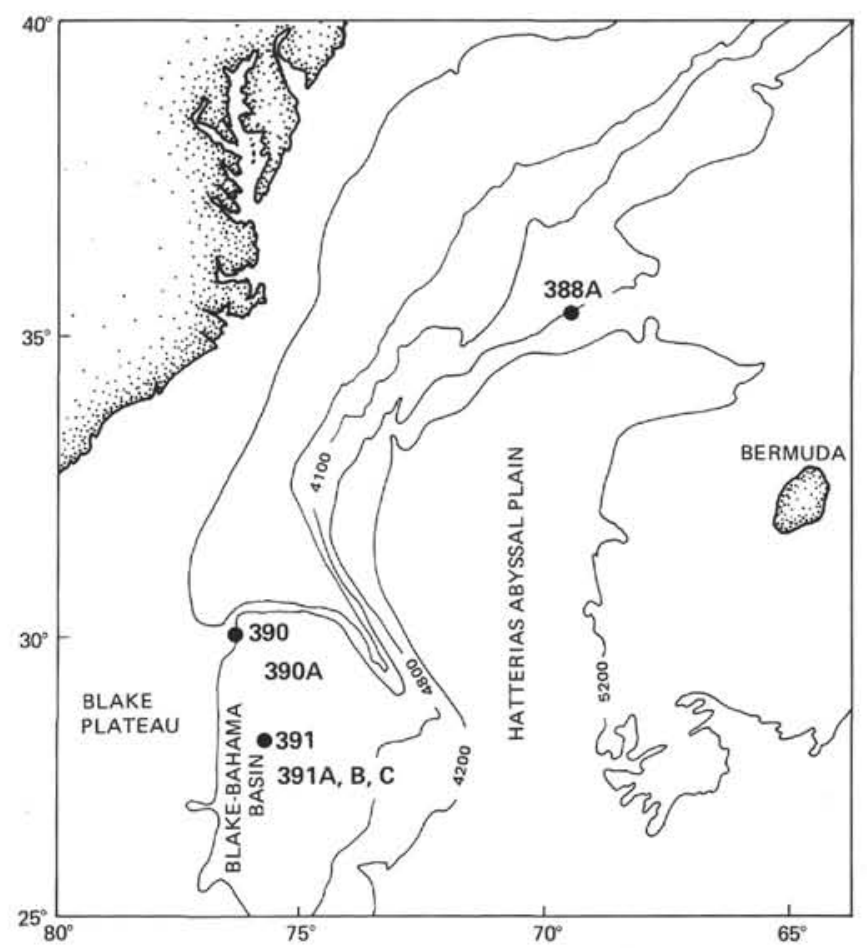

Figure 1. Location of holes from which samples were obtained for geochemical study.

Table 2. Characterization of the lipid fractions is given in Table 3. The $n$-alkane distributions for those cases where sufficient sample was available are given in Figures 4 through 9.

\section{Hole 388A}

The Miocene hemipelagic clays from the lower continental rise hills from Hole $388 \mathrm{~A}$ ranged in subbottom depth from 246 to 332 meters. The sedimentary section is relatively uniform in lithology. Organic content averages $0.97 \pm 0.1$ per cent; carbonate content, less than 0.13 per cent; and organic nitrogen content averages $757 \pm 48 \mathrm{ppm}$ for all but the 298 -meter section which is $2554 \mathrm{ppm}$.

The amount of the total sediment as lipid ranges from $250 \mathrm{ppm}$ for the bottom section then decreases to $50 \mathrm{ppm}$ at the top. The ratio of lipid to total organic carbon is high, namely 2.9 per cent, at the bottom of the unit and is low and constant at $0.7 \pm 0.1$ per cent, for the remainder of the section. The bottom section contained 45.8 per cent of the total lipid as saturates; whereas, the remaining section is highly asphaltic, i.e., 48.4 to 64.5 per cent of the total lipid. 

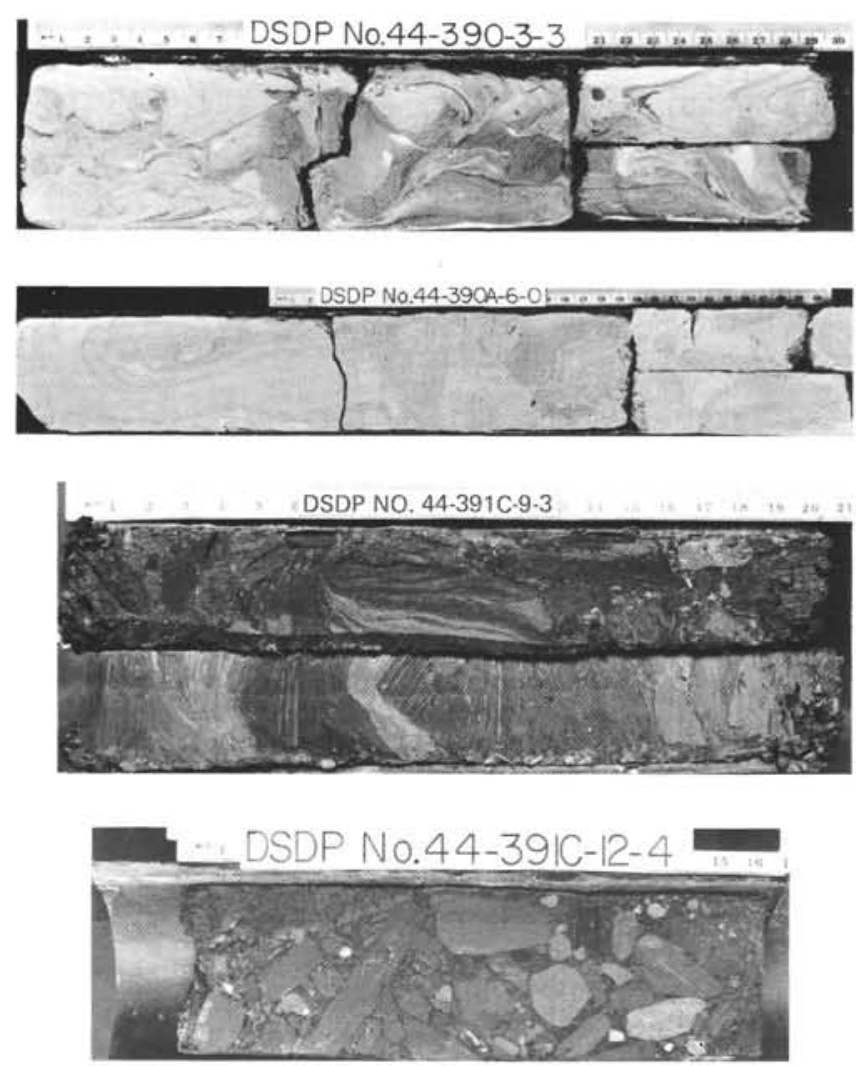

Figure 2. Drilling disturbances observed in DSDP CoreSections 390-3-3, 390A-6-0, 391C-9-3, 391C-12-4.

The carbon isotopic composition of the lipid is relatively constant at $-25.8 \pm 0.6 \delta \mathrm{C}^{13} \mathrm{PDB}$ and is typical of a marine sediment. The carbon isotopic composition of the kerogen is $-23.2 \pm 0.8 \delta \mathrm{C}^{13} \mathrm{PDB}$. The difference between lipid and kerogen is negative and relatively large which suggests a normal sequence of diagenesis.

The ratios of odd to even $n$-alkanes from $n-\mathrm{C}_{23}$ to $n$ $\mathrm{C}_{33}$ as expressed by the $\overline{\mathrm{OEP}}$ value (Scalan and Smith, 1970) for these four Miocene cores are similar, i.e., 2.0 \pm 0.3 , and fall within experimental error. The actual distributions as shown in Figure 4 are similar or identical for all but the 332-meter core (388A-11-0) where the major portion of $n$-alkanes lie in the $n$ - $\mathrm{C}_{15}$ to $n-\mathrm{C}_{25}$ range rather than in the $n-\mathrm{C}_{25}$ to $n-\mathrm{C}_{35}$ range common for the upper part of this unit.

\section{Holes 390 and 390A}

Eight lithologic units were recognized at Site 390 of which one core from each of the following units were studied; the lower Albian unit 7, Maestrichtian unit 5, lower Paleocene unit 4, and Eocene unit 3. All four units have a relatively uniform lithology with an organic content between 0.14 per cent to 0.33 per cent, carbonate carbon content between 6.5 per cent to 8.8 per cent or 54 per cent to 73 per cent as calcium carbonate, ammonium and organic nitrogen content between 47 to $54 \mathrm{ppm}$ and 146 to $153 \mathrm{ppm}$, respectively, lipid carbon to total sediment of $15 \pm 5 \mathrm{ppm}$ and a ratio of lipid carbon to total carbon of 0.34 per cent to 1.0 per cent.
The carbon isotopic composition of the lipid is -27.8 $\delta \mathrm{C}_{\mathrm{PDB}}^{13}$ in the upper Albian sediments to a nearly constant value of $-26.7 \pm 0.3 \delta \mathrm{C}_{\mathrm{PDB}}^{13}$ in the Maestrichtian to Eocene sediments. The isotopic composition of the kerogen varies from -22.9 to $-26.9 \delta \mathrm{C}_{\mathrm{PDB}}^{13}$ with no apparent trends with depth. Because of the small amount of lipid, we are unable to characterize it further.

\section{Holes 391, 391A, 391B, and 391C}

Holes 391, 391A, 391B, and 391C lie in an abyssal area in the Blake-Bahama Basin in the western North Atlantic Ocean. Five lithologic units were recognized from which samples were obtained from each.

Unit 5 consisted of Jurassic (upper Tithonian) claystone and limestone at a depth of 1393 and 1355 meters, respectively. The lower claystone is rich in organic matter with 0.81 per cent organic carbon whereas the limestone section is poor in organic matter with only 0.1 per cent organic carbon. The opposite is true for the proportion of organic carbon present as lipid. The limestone contains 2.15 per cent as opposed to 0.09 per cent in the claystone. The samples in the order of decreasing depth, contained 2.6 per cent and 11.1 per cent carbonate carbon or 21.9 per cent and 92.8 per cent as calcium carbonate, 124 and $42 \mathrm{ppm}$ ammonium nitrogen, 176 and $58 \mathrm{ppm}$ organic nitrogen, and 10 to $20 \mathrm{ppm}$ lipid carbon.

The carbon isotopic composition of these lipid fractions are -27.3 and $-26.5 \delta \mathrm{C}^{13}$ PDB and -22.0 and $20.7 \delta \mathrm{C}^{13}$ PDB for the kerogens.

Unit 4 consisted of upper Tithonian-lower Berriasian to upper Berriasian-lower Valanginian limestones. Five cores were obtained from between 1279 to 1022 meters depth. The bottom of the interval contains very little organic matter; the organic carbon content is 0.2 per cent which increases to 1.1 per cent at the top of the unit. The sample contained 9.7 per cent to 6.0 per cent carbonate carbon or from 80.6 per cent to 49.8 per cent as calcium carbonate, 40 to $97 \mathrm{ppm}$ ammonium nitrogen, and 51 to $303 \mathrm{ppm}$ organic nitrogen with the organic nitrogen increasing to the top of the section. The lipid carbon increases from a low of $10 \mathrm{ppm}$ at the bottom of the unit to $790 \mathrm{ppm}$ at 1185 meters, then decreases to $50 \mathrm{ppm}$ at the top of the unit. The ratio of lipid to total organic carbon is low at the bottom of the unit, namely 0.62 per cent, increases to a high of 23.5 per cent at 1234 meters, then decreases steadily to 0.41 per cent at the top of the interval.

The isotopic composition of the lipid fractions is steadily lighter from the bottom to the top of the unit from -25.6 to $-29.1 \delta \mathrm{C}^{13} \mathrm{PDB}$. The kerogen isotopic composition varies from a low of -21.6 to a high of $26.3 \delta \mathrm{C}^{13}$ PDB with no trends with depth.

Enough lipid was obtained from core-sections 391C$35-4,391 \mathrm{C}-30-2$, and $391 \mathrm{C}-26-3$ for its full characterization. As shown in Table 3, all three sections were similar in their lipid properties. The saturate, aromatic, and asphaltic fractions averaged 2.8, 84.7, and 12.5 per cent respectively. The isotopic composition of the aromatic and asphaltic fractions averaged $-26.8 \pm 0.6 \delta \mathrm{C}^{13} \mathrm{PDB}$. The atomic ration of hydrogen to carbon is $1.64 \pm 0.02$. 
TABLE 1

Lithology of Core-Sections From DSDP Leg 44

Description and Carbonate and Organic Carbon Content of Different Textural Types

\begin{tabular}{|c|c|c|c|c|c|}
\hline \multirow[b]{2}{*}{ Hole-Core-Section } & \multirow[b]{2}{*}{ Lithological Description } & \multirow[b]{2}{*}{$\begin{array}{c}\text { Textural } \\
\text { Unit }^{\mathrm{a}}\end{array}$} & \multicolumn{2}{|c|}{ Carbonate as } & \multirow{2}{*}{$\begin{array}{l}\text { Organic } \\
\text { Carbon } \\
\text { (wt. \%) }\end{array}$} \\
\hline & & & $\begin{array}{l}\text { Carbon } \\
\text { (wt. \%) }\end{array}$ & $\begin{array}{l}\mathrm{CaCO}_{3} \\
\text { (wt. \%) }\end{array}$ & \\
\hline $388 \mathrm{~A}-5-0$ & $\begin{array}{l}\text { Clay, dark greenish gray (5Gy } 4 / 1,5 \mathrm{G} 4 / 1) \text {, } \\
\text { faintly mottled, stiff (unit no. 1); contains } \\
\text { hard sideritic (?) concentrations (burrow } \\
\text { fillings [?]), concentrated in indistinct } \\
\text { layers (units } 2 \text { and } 3 \text { ), some with soft } \\
\text { microcrystalline sideritic (?) material; } \\
\text { pyrite is common; composition } 65 \%-90 \% \\
\text { clay minerals, } 10 \%-35 \% \text { quartz, up to } 10 \% \\
\text { mica, and traces of feldspar }\end{array}$ & $\begin{array}{l}1 \\
2 \\
3\end{array}$ & $\begin{array}{l}<0.1 \\
<0.1 \\
<0.1\end{array}$ & $\begin{array}{l}<0.83 \\
<0.83 \\
<0.83\end{array}$ & $\begin{array}{l}1.13 \\
0.53 \\
0.99\end{array}$ \\
\hline $388 A-7-3$ & $\begin{array}{l}\text { Clay, dark greenish gray ( } 5 \mathrm{G} 4 / 1) \text {, faintly } \\
\text { mottled, stiff, with rare siderite (?) } \\
\text { concretions, composition } 85 \%-95 \% \text { clay } \\
\text { minerals, } 5 \%-15 \% \text { quartz, traces of heavy } \\
\text { minerals (rutite), chlorite, carbonate } \\
\text { rhombs, chert, benthic foraminifers, and } \\
\text { calcareous nannofossils }\end{array}$ & 1 & $<0.1$ & $<0.83$ & 1.02 \\
\hline $388 \mathrm{~A}-7-4$ & $\begin{array}{l}\text { Clay, dark greenish gray (5GY4/1), faintly } \\
\text { mottled, stiff with rare siderite (?) } \\
\text { concretions, composition } 85 \%-95 \% \text { clay } \\
\text { minerals, } 5 \%-15 \% \text { quartz, traces of heavy } \\
\text { minerals (rutite), chlorite, carbonate } \\
\text { rhombs, chert, benthic foraminifers, and } \\
\text { calcareous nannofossils }\end{array}$ & 1 & $<0.1$ & $<0.83$ & 1.08 \\
\hline $388 \mathrm{~A}-11-0$ & $\begin{array}{l}\text { Clay, grayish olive (10Y4/2) (unit no. 1) } \\
\text { soft, plastic with dusky red (5R3/4) } \\
\text { siderite on black (N1) py rite (?) } \\
\text { concretions (unit no. } 2 \text { ) scattered through- } \\
\text { out sediment; sediment is badly disturbed } \\
\text { in first } 13 \mathrm{~cm} \text { (unit a) and partially } \\
\text { disturbed in the remaining } 24 \mathrm{~cm} \text { (units b } \\
\text { and c) with mixture of soft grayish orange } \\
(20 \mathrm{YR} 7 / 4 \text { ) (unit no. } 3 \text { ) and firm fragments } \\
\text { of drilling slurry; composition } 80 \%-90 \% \\
\text { clay minerals, } 5 \%-10 \% \text { quartz, and traces of } \\
\text { feldspar, mica, heavy minerals, chlorite, } \\
\text { zeolites, carbonate rhombs, and calcareous } \\
\text { nannofossils }\end{array}$ & $\begin{array}{l}1 \\
2 \\
3\end{array}$ & $\begin{array}{c}<0.1 \\
0.58 \\
7.54\end{array}$ & $\begin{array}{r}<0.83 \\
4.83 \\
62.83\end{array}$ & $\begin{array}{l}1.00 \\
1.42 \\
0.48\end{array}$ \\
\hline $390-3-3$ & $\begin{array}{l}\text { Badly disturbed marly nannofossil ooze, } \\
\text { pale blue-green ( } 5 \mathrm{BG} 7 / 2 \text { ) (unit no. } 1 \text { ); } \\
\text { considerable mixing due to drilling of a } \\
\text { marly nannofossil ooze, light reddish brown } \\
\text { (5YR6/4) (unit no. 2) with wisps of light } \\
\text { brown (5YR5/6) (unit no. } 3 \text { ) admixed }\end{array}$ & $\begin{array}{l}1 \\
2 \\
3\end{array}$ & $\begin{array}{l}7.36 \\
6.95 \\
6.84\end{array}$ & $\begin{array}{l}61.33 \\
57.92 \\
57.00\end{array}$ & $\begin{array}{l}0.25 \\
0.32 \\
0.37\end{array}$ \\
\hline $390 A-6-0$ & $\begin{array}{l}\text { Nannofossil ooze, light yellowish brown } \\
\text { (10YR6/4) (unit no. 1) with very pale } \\
\text { brown (10YR8/3 to 10YR8/4) (unit no. 2) } \\
\text { banding due to drilling; composition } 70 \% \\
\text { calcareous nannofossils, } 10 \% \text { planktonic } \\
\text { foraminifers, } 3 \% \text { benthic foraminifers, } \\
5 \% \text { clay minerals, } 5 \% \text { zeolites, } 5 \% \text { unidenti- } \\
\text { fied minerals }\end{array}$ & $\begin{array}{l}1 \\
2\end{array}$ & $\begin{array}{l}7.92 \\
8.51\end{array}$ & $\begin{array}{l}66.00 \\
70.92\end{array}$ & $\begin{array}{l}0.23 \\
0.29\end{array}$ \\
\hline $390 \mathrm{~A}-10-0$ & $\begin{array}{l}\text { Marly nannofossil ooze }(20 \mathrm{YR} 8 / 2) \text { (unit } \\
\text { no. 1) with } 1 \mathrm{~mm} \text { - to } 8 \mathrm{~mm} \text {-size dark } \\
\text { olive-gray }(5 \mathrm{Y} 3 / 2) \text { to dark gray (N3) clay } \\
\text { filled burrows (unit no. } 2 \text { ) }\end{array}$ & $\begin{array}{l}1 \\
2\end{array}$ & $\begin{array}{l}7.90 \\
7.24\end{array}$ & $\begin{array}{l}65.83 \\
60.33\end{array}$ & $\begin{array}{l}0.26 \\
0.29\end{array}$ \\
\hline $390 \mathrm{~A}-12-0$ & $\begin{array}{l}\text { Nannofossil ooze, light bluish gray }(5 \mathrm{~B} 7 / 1) \text {, } \\
\text { homogeneous; composition } 55 \%-75 \% \\
\text { calcareous nannofossils, } 15 \% \text { unspecified } \\
\text { carbonate, } 25 \% \text { clay minerals, } 5 \% \text { quartz, } \\
2 \% \text { zeolites, traces of mica, volcanic glass, } \\
\text { heavy minerals, benthic foraminifers, radio- } \\
\text { larians, sponge spicules }\end{array}$ & 1 & 8.45 & 70.41 & 0.23 \\
\hline
\end{tabular}




\begin{tabular}{|c|c|c|c|c|c|}
\hline \multirow[b]{2}{*}{ Hole-Core-Section } & \multirow[b]{2}{*}{ Lithological Description } & \multirow[b]{2}{*}{$\begin{array}{c}\text { Textural } \\
\text { Unita }\end{array}$} & \multicolumn{2}{|c|}{ Carbonate as } & \multirow{2}{*}{$\begin{array}{l}\text { Organic } \\
\text { Carbon } \\
\text { (wt. \%) }\end{array}$} \\
\hline & & & $\begin{array}{l}\text { Carbon } \\
\text { (wt. \%) } \\
\end{array}$ & $\begin{array}{l}\mathrm{CaCO}_{3} \\
\text { (wt.\%) }\end{array}$ & \\
\hline $391-1-2$ & $\begin{array}{l}\text { Silty hemipelagic clay, soft, olive-gray } \\
(5 \mathrm{Y} 4 / 1) \text { (unit no. 1) with small olive-gray } \\
(5 \mathrm{Y} 3 / 2) \text { concretions (unit no. } 2) \text {; clay } \\
\text { unit from } 3 \text { to } 6 \mathrm{~cm} \text { from top of core, soft } \\
\text { pale yellowish brown }(10 \mathrm{YR} 6 / 2) \text { (unit no. } 3 \text { ) }\end{array}$ & $\begin{array}{l}1 \\
2 \\
3\end{array}$ & $\begin{array}{l}0.51 \\
1.24 \\
5.71\end{array}$ & $\begin{array}{r}4.25 \\
10.33 \\
47.58\end{array}$ & $\begin{array}{l}1.30 \\
1.27 \\
0.84\end{array}$ \\
\hline $391 \mathrm{~A}-3-4$ & $\begin{array}{l}\text { Calcareous "silt", yellowish gray; }(5 Y 8 / 1) \text {, } \\
\text { homogeneous, stiff }\end{array}$ & 1 & 11.34 & 94.50 & 0.15 \\
\hline $391 \mathrm{~A}-4-3$ & $\begin{array}{l}\text { Chalk, light bluish gray }(5 \mathrm{Y} 7 / 1,5 \mathrm{Y} 7 / 2) \\
\text { (unit no. 1) with light gray }(5 \mathrm{Y} 7 / 2) \\
\text { (unit no. 2) mottling layers, homogeneous }\end{array}$ & $\begin{array}{l}1 \\
2\end{array}$ & $\begin{array}{l}10.56 \\
10.84\end{array}$ & $\begin{array}{l}88.00 \\
90.33\end{array}$ & $\begin{array}{l}0.29 \\
0.23\end{array}$ \\
\hline $391 \mathrm{~A}-5-0$ & $\begin{array}{l}\text { Marly chalk pale olive }(10 \mathrm{Y} 6 / 2) \text { (unit no. } 1 \text { ) } \\
\text { with } 1 \mathrm{~mm} \text { to } 10 \mathrm{~mm} \text { size dark greenish gray } \\
(5 \mathrm{G} 4 / 1,5 \mathrm{G} 4 / 1) \text { and olive-gray ( } 5 \mathrm{Y} 4 / 1) \\
\text { clasts (unit no. } 2 \text { ) ; clasts are mostly green } \\
\text { siliceous clay; composition (chalk) } 50 \% \\
\text { unspecified carbonate, } 15 \% \text { calcareous } \\
\text { nannofossils, } 35 \% \text { clay minerals, traces } \\
\text { quartz, feldspar, mica, dolomite, siliceous } \\
\text { microfossils }\end{array}$ & $\begin{array}{l}1 \\
2\end{array}$ & $\begin{array}{l}9.34 \\
2.51\end{array}$ & $\begin{array}{l}77.83 \\
20.92\end{array}$ & $\begin{array}{l}0.55 \\
1.48\end{array}$ \\
\hline $391 \mathrm{~A}-6-3$ & $\begin{array}{l}\text { Marly chalk, yellowish gray }(5 \mathrm{Y} 7 / 2) \text { (unit } \\
\text { no. 1) with small ( } 3 \mathrm{~mm} \text { ) greenish gray } \\
(5 \mathrm{GY} 4 / 1) \text { (unit no. } 2) \text { clasts; composition } \\
40 \% \text { calcareous nannofossils, } 35 \% \text { unspeci- } \\
\text { fied carbonates, } 3 \% \text { foraminifers, } 19 \% \text { clay } \\
\text { minerals, } 3 \% \text { sponge spicules, traces of } \\
\text { quartz and radiolarians }\end{array}$ & $\begin{array}{l}1 \\
2\end{array}$ & $\begin{array}{r}10.54 \\
6.44\end{array}$ & $\begin{array}{l}87.83 \\
53.67\end{array}$ & $\begin{array}{l}0.30 \\
0.90\end{array}$ \\
\hline $391 \mathrm{~A}-9-0$ & $\begin{array}{l}\text { Siliceous muddy chalk and marly chalk, } \\
\text { pale olive }(10 \mathrm{Y} 6 / 2) \text { (unit no. } 1) \text { with } 1 \text { to } \\
20 \text {-mm size greenish gray }(5 \mathrm{Y} 3 / 2) \text { (unit } \\
\text { no. } 2 \text { ) clay clasts; most clasts are elongated } \\
\text { parallel to bedding; composition } 15 \% \\
\text { calcareous nannofossils, } 20 \% \text { unspecified } \\
\text { carbonate, } 30 \% \text { radiolarians, } 35 \% \text { clay } \\
\text { minerals, traces of foraminifers and sponge } \\
\text { spicules }\end{array}$ & $\begin{array}{l}1 \\
2\end{array}$ & $<0.1$ & $\begin{array}{l}<0.83 \\
71.67\end{array}$ & $\begin{array}{l}1.85 \\
0.46\end{array}$ \\
\hline $391 \mathrm{~A}-10-0$ & $\begin{array}{l}\text { Marly chalk, pale olive }(10 \mathrm{Y} 6 / 2) \text { (unit no. } \\
\text { 1) with } 1 \mathrm{~mm} \text { to } 7 \mathrm{~mm} \text { size greenish gray } \\
\text { (5G6/1) and olive-gray }(5 \mathrm{Y} 3 / 1) \text { clay } \\
\text { clasts (unit no. } 2) \text {; mosts clasts are elon- } \\
\text { gated parallel to bedding; composition } \\
45 \% \text { calcareous nannofossils, } 20 \% \text { unspeci- } \\
\text { fied carbonate, } 30 \% \text { clay minerals, } 3 \% \\
\text { siliceous microfossils, trace foraminifers }\end{array}$ & $\begin{array}{l}1 \\
2\end{array}$ & $\begin{array}{l}8.43 \\
0.97\end{array}$ & $\begin{array}{r}70.25 \\
8.08\end{array}$ & $\begin{array}{l}0.48 \\
1.40\end{array}$ \\
\hline $391 \mathrm{~A}-11-0$ & $\begin{array}{l}\text { Marly chalk (unit no. } 1 \text { ), greenish gray } \\
(5 \mathrm{GY} 6 / 1) \text { to pale olive-gray (10Y6/2) } \\
\text { with } 1 \mathrm{~mm} \text { - to } 15 \mathrm{~mm} \text {-sized greenish gray } \\
\text { and olive-gray (5Y3/1) clay clasts (unit } \\
\text { no. } 2 \text { ); mosts clasts are elongated parallel } \\
\text { to bedding; composition } 30 \% \text { calcareous } \\
\text { nannofossils, } 25 \% \text { unspecified carbonate, } \\
6 \% \text { foraminifers, } 25 \% \text { clay minerals, } 10 \% \\
\text { sponge spicules, } 3 \% \text { radiolarians, traces of } \\
\text { quartz, pyrite, zeolites }\end{array}$ & $\begin{array}{l}1 \\
2\end{array}$ & $\begin{array}{l}8.64 \\
1.50\end{array}$ & $\begin{array}{l}72.00 \\
12.50\end{array}$ & $\begin{array}{l}0.43 \\
1.46\end{array}$ \\
\hline $391 \mathrm{~A}-12-0$ & $\begin{array}{l}\text { Marly chalk (unit no. } 1 \text { ), pale olive } \\
(10 \mathrm{Y} 6 / 2) \text { with } 1 \mathrm{~mm}-\text { to } 13 \mathrm{~mm} \text {-size } \\
\text { moderate olive-brown }(5 \mathrm{Y} 4 / 4) \text {, olive-gray } \\
(5 \mathrm{Y} 3 / 2) \text {, pinkish gray }(5 \mathrm{YR} 8 / 1) \text {, and light } \\
\text { bluish gray ( } 5 \mathrm{~B} 7 / 1) \text { clay clasts (unit no. } 2 \text { ) }\end{array}$ & $\begin{array}{l}1 \\
2\end{array}$ & $\begin{array}{l}9.92 \\
0.90\end{array}$ & $\begin{array}{r}82.67 \\
7.50\end{array}$ & $\begin{array}{l}0.29 \\
1.16\end{array}$ \\
\hline $391 \mathrm{~A}-13-0$ & Mudstone, olive-gray $(5 \mathrm{Y} 4 / 2)$, homogeneous & 1 & 1.43 & 11.92 & 2.71 \\
\hline $391 \mathrm{~A}-20-2$ & $\begin{array}{l}\text { Claystone (unit no. 1) banded dark olive } \\
(5 \mathrm{Y} 3 / 2) \text { and dark greenish gray ( } 5 \mathrm{G} 4 / 1) \text {, } \\
\text { fissile; well-defined burrows (unit nos. } 2 \\
\text { and } 3 \text { ); composition } 95 \% \text { clay minerals, } \\
1 \% \text { sponge spicules, traces of quartz, mica, } \\
\text { pyrite, unspecified carbonate, radiolarians }\end{array}$ & $\begin{array}{l}1 \\
2 \\
3\end{array}$ & $\begin{array}{l}<0.1 \\
<0.1 \\
<0.1\end{array}$ & $\begin{array}{l}<0.83 \\
<0.83 \\
<0.83\end{array}$ & $\begin{array}{l}1.87 \\
1.56 \\
1.31\end{array}$ \\
\hline
\end{tabular}


TABLE 1 - Continued

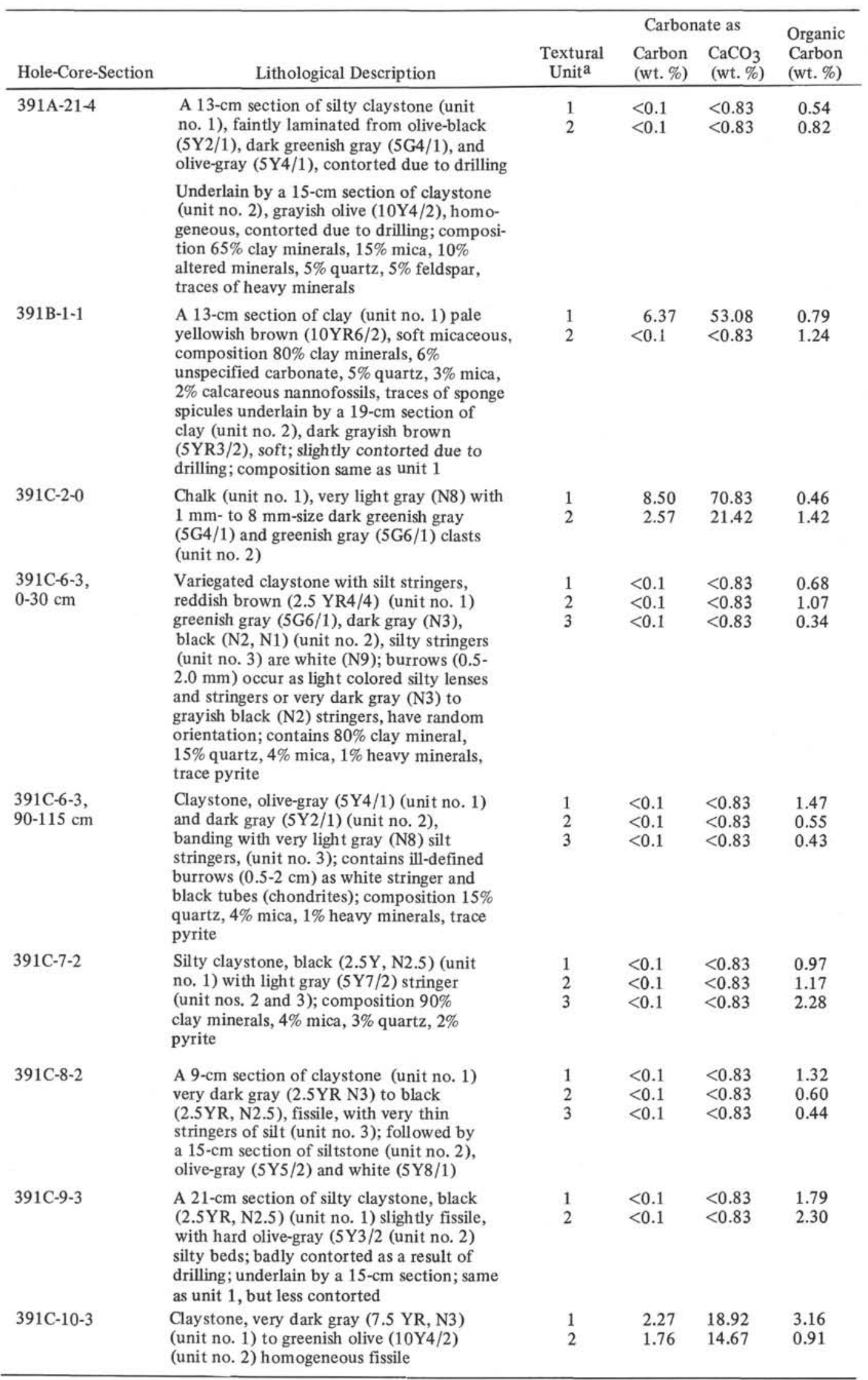




\begin{tabular}{|c|c|c|c|c|c|}
\hline \multirow[b]{2}{*}{ Hole-Core-Section } & \multirow[b]{2}{*}{ Lithological Description } & \multirow[b]{2}{*}{$\begin{array}{l}\text { Textural } \\
\text { Unit }^{\mathrm{a}}\end{array}$} & \multicolumn{2}{|c|}{ Carbonate as } & \multirow{2}{*}{$\begin{array}{l}\text { Organic } \\
\text { Carbon } \\
\text { (wt. \%) }\end{array}$} \\
\hline & & & $\begin{array}{l}\text { Carbon } \\
\text { (wt. \%) }\end{array}$ & $\begin{array}{l}\mathrm{CaCO}_{3} \\
\text { (wt. \%) }\end{array}$ & \\
\hline $391 C-11-2$ & $\begin{array}{l}\text { Silty calcareous claystone, olive gray ( } 5 \text { Y } 3 / 2) \\
\text { (unit no. } 1 \text { ), light olive }(5 \text { Y } 5 / 2) \text {, greenish } \\
\text { gray (5GY6/1), and olive-black (5Y } 2 / 1 \text { ) } \\
\text { (unit no. 2) laminated, composition } 84 \% \\
\text { clay minerals, } 10 \% \text { calcareous nannofossils, } \\
4 \% \text { mica, } 1 \% \text { quartz, } 1 \% \text { heavy minerals }\end{array}$ & $\begin{array}{l}1 \\
2\end{array}$ & $\begin{array}{l}7.20 \\
8.69\end{array}$ & $\begin{array}{l}60.00 \\
72.42\end{array}$ & $\begin{array}{l}0.51 \\
0.50\end{array}$ \\
\hline $391 C-12-4$ & $\begin{array}{l}\text { Claystone, grayish black (N2) (unit no. } 1 \text { ) } \\
\text { with moderate brown (5YR4/4) (unit no. } 2 \text { ), } \\
\text { olive-gray ( } 5 \text { Y3/2) (unit no. } 3 \text { ), and light } \\
\text { gray (N7) unit no. } 4 \text { ) mottling; badly } \\
\text { disturbed by drilling }\end{array}$ & $\begin{array}{l}1 \\
2 \\
3 \\
4\end{array}$ & $\begin{array}{c}<0.1 \\
<0.1 \\
5.30 \\
10.80\end{array}$ & $\begin{array}{l}<0.83 \\
<0.83 \\
44.17 \\
90.00\end{array}$ & $\begin{array}{l}1.45 \\
0.49 \\
0.95 \\
0.46\end{array}$ \\
\hline $391 C-16-1$ & $\begin{array}{l}\text { Limestone with shale partings and sandy } \\
\text { siltstone beds, gray }(5 \mathrm{Y} 5 / 1) \text { to olive gray } \\
(5 \mathrm{Y} 5 / 2) \text { to black }(5 \mathrm{Y} 2.5 / 1) \text {; burrowing } \\
\text { bioturbation, cross laminations; composition } \\
73 \% \text { clay minerals, } 15 \% \text { calcareous nanno- } \\
\text { fossils, } 5 \% \text { unspecified carbonate, } 2 \% \text { quartz, } \\
2 \% \text { mica, } 2 \% \text { pyrite, } 1 \% \text { feldspar, trace } \\
\text { heavy minerals }\end{array}$ & 1 & 2.62 & 21.83 & 0.97 \\
\hline $391 C-26-3$ & $\begin{array}{l}\text { A } 3-\mathrm{cm} \text { of limestone, light gray (N7), } \\
\text { laminated (unit no. } 1 \text { ). Underlain by a 2-cm } \\
\text { section of limestone, olive-gray ( } 5 \text { Y } 5 / 2 \text { ) } \\
\text { (unit no. 2) with shale laminae; some } \\
\text { burrowing olive black }(5 Y 2 / 1) \text {; then a } 3-\mathrm{cm} \\
\text { section of unit no. } 1 .\end{array}$ & $\begin{array}{l}1 \\
2 \\
3\end{array}$ & $\begin{array}{l}7.45 \\
5.68 \\
2.85\end{array}$ & $\begin{array}{l}62.08 \\
47.33 \\
23.75\end{array}$ & $\begin{array}{l}0.62 \\
0.89 \\
0.56\end{array}$ \\
\hline $391 C-30-2$ & $\begin{array}{l}\text { A } 5 \text {-cm section of laminated clayey lime- } \\
\text { stone (unit no. } 1) \text {, light gray (5Y6/1) } \\
\text { to light olive-gray ( } 5 \mathrm{Y} 5 / 2) \text { with dark } \\
(1 \mathrm{~mm} \text { ) laminae of olive-gray }(5 \mathrm{Y} 3 / 2) \\
\text { claystone; underlain by a } 1 \text {-cm section of } \\
\text { claystone (unit no. } 2) \text {, olive-gray }(5 \mathrm{Y} 3 / 2) \text {, } \\
\text { fissile, } 10 \mathrm{~mm} \text { band }\end{array}$ & $\begin{array}{l}1 \\
2\end{array}$ & $\begin{array}{l}7.67 \\
1.84\end{array}$ & $\begin{array}{l}63.92 \\
15.33\end{array}$ & $\begin{array}{l}0.90 \\
1.22\end{array}$ \\
\hline $391 C-35-4$ & $\begin{array}{l}\text { Clayey limestone (unit no. 1), light bluish } \\
\text { gray (5B } 7 / 1 \text { ) to light gray (N7); with } 1 \mathrm{~mm} \\
\text { to } 10 \mathrm{~mm} \text { olive-gray (5Y3/2) clay clasts } \\
\text { (unit no. } 2 \text { ); composition } 50 \% \text { clay } \\
\text { minerals, 20\%-30\% unspecified carbonate, } \\
20 \%-30 \% \text { calcareous nannofossils, trace } \\
\text { quartz, feldspar, mica, pyrite }\end{array}$ & $\begin{array}{l}1 \\
2\end{array}$ & $\begin{array}{l}7.67 \\
4.22\end{array}$ & $\begin{array}{l}63.92 \\
35.17\end{array}$ & $\begin{array}{l}0.35 \\
2.40\end{array}$ \\
\hline $391 C-40-2$ & $\begin{array}{l}\text { Limestone (unit no. } 1) \text {, white }(7.5 \mathrm{YR}, \mathrm{N} 8 \text { ) } \\
\text { with dark greenish }(5 \mathrm{G} 4 / 1) \text { very irregular } \\
\text { clay stringers (unit no. } 2 \text { ); calcispheres } \\
\text { (calcified radiolarians [?]) }\end{array}$ & $\begin{array}{l}1 \\
2\end{array}$ & $\begin{array}{r}10.06 \\
4.70\end{array}$ & $\begin{array}{l}83.83 \\
39.17\end{array}$ & $\begin{array}{l}0.07 \\
0.44\end{array}$ \\
\hline $391 C-48-2$ & $\begin{array}{l}\text { Limestone, pale yellowish brown }(10 \mathrm{YR} 6 / 2) \text {, } \\
\text { mottled to pale brown }(5 \mathrm{YR} 5 / 2) \text {, homo- } \\
\text { geneous, hard }\end{array}$ & 1 & $\mathrm{~T} / \mathrm{S}$ & $\mathrm{T} / \mathrm{S}$ & $\mathrm{T} / \mathrm{S}$ \\
\hline \multirow[t]{3}{*}{$391 C-52-2$} & $\begin{array}{l}\text { A } 9-\mathrm{cm} \text { section of claystone, dark reddish } \\
\text { brown }(5 \mathrm{YR} 3 / 2) \text { (unit no. } 1) \text {; underlain by } \\
\text { a 3-cm section of claystone (unit no. } 2) \\
\text { greenish gray }(5 \mathrm{G} 6 / 1) \text { to dark greenish gray } \\
(5 \mathrm{G} 4 / 1) \text { banded }\end{array}$ & $\begin{array}{l}1 \\
2\end{array}$ & $\begin{array}{l}2.87 \\
2.78\end{array}$ & $\begin{array}{l}23.92 \\
23.17\end{array}$ & $\begin{array}{l}0.73 \\
0.77\end{array}$ \\
\hline & $\begin{array}{l}\text { Greenish gray claystone is identical to } \\
\text { reddish brown claystone except for color; } \\
\text { composition } 65 \% \text { clay minerals, } 15 \% \text { cal- } \\
\text { careous nannofossils, } 8 \% \text { iron oxides, } 3 \% \\
\text { quartz, } 3 \% \text { mica, } 5 \% \text { unspecified carbonate, } \\
\text { trace heavy minerals, and volcanic glass are } \\
\text { common; sediment is bioturbated; micro- } \\
\text { stylobites present }\end{array}$ & & & & \\
\hline & $\begin{array}{l}\text { Unit B-shale, grayish olive-green }(10 \mathrm{Y} 4 / 2) \text {, } \\
\text { fissile }\end{array}$ & & & & \\
\hline
\end{tabular}

\footnotetext{
${ }^{\mathrm{a}}$ As noted in the sampling and study procedures section, textural units refer to physically different units noted on the millled half section of the core. These units are noted in the Lithological Descriptions. For homogeneous cores, chips taken for organic and carbonate carbon analyses were taken from the center of the core.
} 


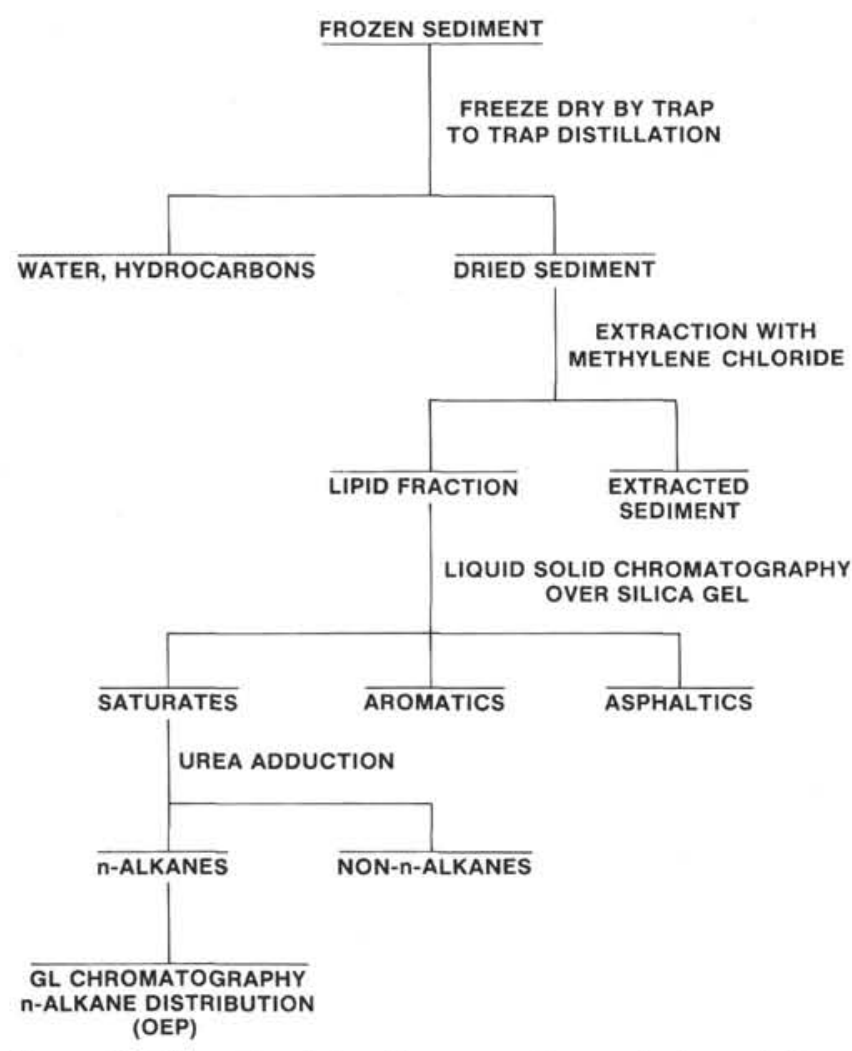

Figure 3. Flow sheet for the separation and characterization of organic matter from DSDP cores from Leg 44.

The $n$-alkane distributions for these three sections are shown in Figure 5. These distributions show a progressive increase in the smooth $n$-alkane maximum at $\mathrm{n}-\mathrm{C}_{27}$ with depth. A typical recent sediment distribution with an odd-carbon preference and an oddcarbon maximum at $n-\mathrm{C}_{31}$ is observed in the distribution for Section 391C-26-3. Section 391C-30-2 contains a bimodal $n$-alkane distribution with a relatively smooth maximum at $n-\mathrm{C}_{27}$ and the oddcarbon preference maximum at $n-C_{31}$. Section 391C-354 contains only the relatively smooth maximum at $n$ $\mathrm{C}_{27}$. The $\overline{\mathrm{OEP}}$ values are indicative of cores in an intermediate and final stage of source rock development.

Unit 3 penetrated the Lower and Upper Cretaceous sections. Nine cores of olive-gray to grayish black silty clays and claystones were obtained from a depth interval of 959 to 655 meters. The geochemical properties of the sample are given in Table 2. The organic carbon content is relatively high throughout most of this unit averaging 1.1 per cent and ranging from a high of 2 per cent near the bottom of the unit to a low of 0.3 per cent at 655 meters. The carbonate carbon content is low at the bottom of the unit, that is, less than 0.8 per cent as calcium carbonate, increases to a high of 64.2 per cent at 927 meters, then decreases to less than 0.8 per cent at the 692 -meter section, then increases to 70.9 per cent at 656 meters. The ammonium nitrogen value varies from 49 to $169 \mathrm{ppm}$ with no trends with depth. The organic nitrogen ranges from 282 to $707 \mathrm{ppm}$ with the samples rich in organic matter containing slightly more organic nitrogen.
The carbon isotopic composition values for the lipid fractions of the 959 to 727 meter interval are similar, i.e., $-28.5 \pm 0.4 \delta \mathrm{C}_{\mathrm{PDB}}^{13}$ with the exception of the 897.6meter interval which is one del unit lighter than this average. The upper three core sections are also similar, is, -26.8 $\pm 0.3 \delta \mathrm{C}_{\mathrm{PDB}}^{13}$. Carbon isotopic composition values of the kerogen are typically heavier than the lipid and range randomly from -21.9 to $-25.9 \delta \mathrm{C}_{\mathrm{PDB}}^{13}$. The algebraic difference between lipid and kerogen is large and negative for the entire unit suggesting a normal sequence of diagenesis.

Enough lipid was available from four of the nine core sections for a complete lipid analysis. The sediments toward the bottom of the unit contained slightly more saturates and a larger asphaltic fraction than the upper part. As shown in Table 3, the carbon isotopic composition of the aromatic and asphaltic fractions of the lower portion of this unit is lighter than in the upper portion.

Elemental analysis for carbon, hydrogen, nitrogen, and sulfur was completed on the lipid fraction from Sections 391-10-3 and 391-6-3. Lipid from Section 10-3 contained more elemental carbon and less oxygen than Section 6-3. However, the ratio of hydrogen to carbon lipids is within experimental error, that is, $1.64 \pm 0.001$.

The $n$-alkane distribution curves for the four core sections studies are shown in Figure 6. The general shapes of the various curves are the same. As shown in Table 3 , the $\overline{\mathrm{OEP}}$ values for these sections vary randomly with slightly higher values toward the bottom of the unit.

Unit 2 consisted of Miocene intraclastic chalk from 642 to 151 meters. Eleven samples were obtained from this unit from Holes 391A and 391C. The lithologies ranged from predominantly chalk to mudstone and claystone. The organic carbon content for eight of the eleven cores fell at or below 0.5 per cent. The carbonate content is relatively high averaging $8.9 \pm 2.1$ per cent carbonate carbon or $74 \pm 15$ per cent as calcium carbonate for all but the deepest lying sample, 391A-202 , which contained less than 0.1 per cent carbonate carbon. The ammonium nitrogen content for the Miocene interval of Hole 391A decreases from the bottom to top of the unit from a high of $220 \mathrm{ppm}$ to a low of $79 \mathrm{ppm}$. The organic nitrogen content for Hole 391A generally decreases from the oldest to the youngest sediments within this unit: from a high of 847 $\mathrm{ppm}$ to a low of $25 \mathrm{ppm}$. The ammonium and organic nitrogen content of Section 391C-2-0 is more like those values from unit 4 within Hole $391 \mathrm{C}$ than those of unit 3 from Hole 391A.

The concentration of lipid carbon relative to the dry weight of sample averages $120 \mathrm{ppm}$ and ranges from a low of $20 \mathrm{ppm}$ to a high of $450 \mathrm{ppm}$. The portion of organic carbon as lipid carbon averages 2.1 per cent with a range of 0.57 to 4.08 per cent. No trends in either the lipid carbon to total sediment or total organic carbon are observed with depth.

The average value of the saturate, aromatic, and asphaltic fractions in weight percent and where possible the corresponding carbon isotopic composition values in $\delta \mathrm{C}^{13} \mathrm{PDB}$ units for these sediments are 14, $27(-24.9)$, 
TABLE 2

Chemistry of Core-Sections From DSDP Leg 44 Geologic Age, Depth, Elemental and Isotopic Composition

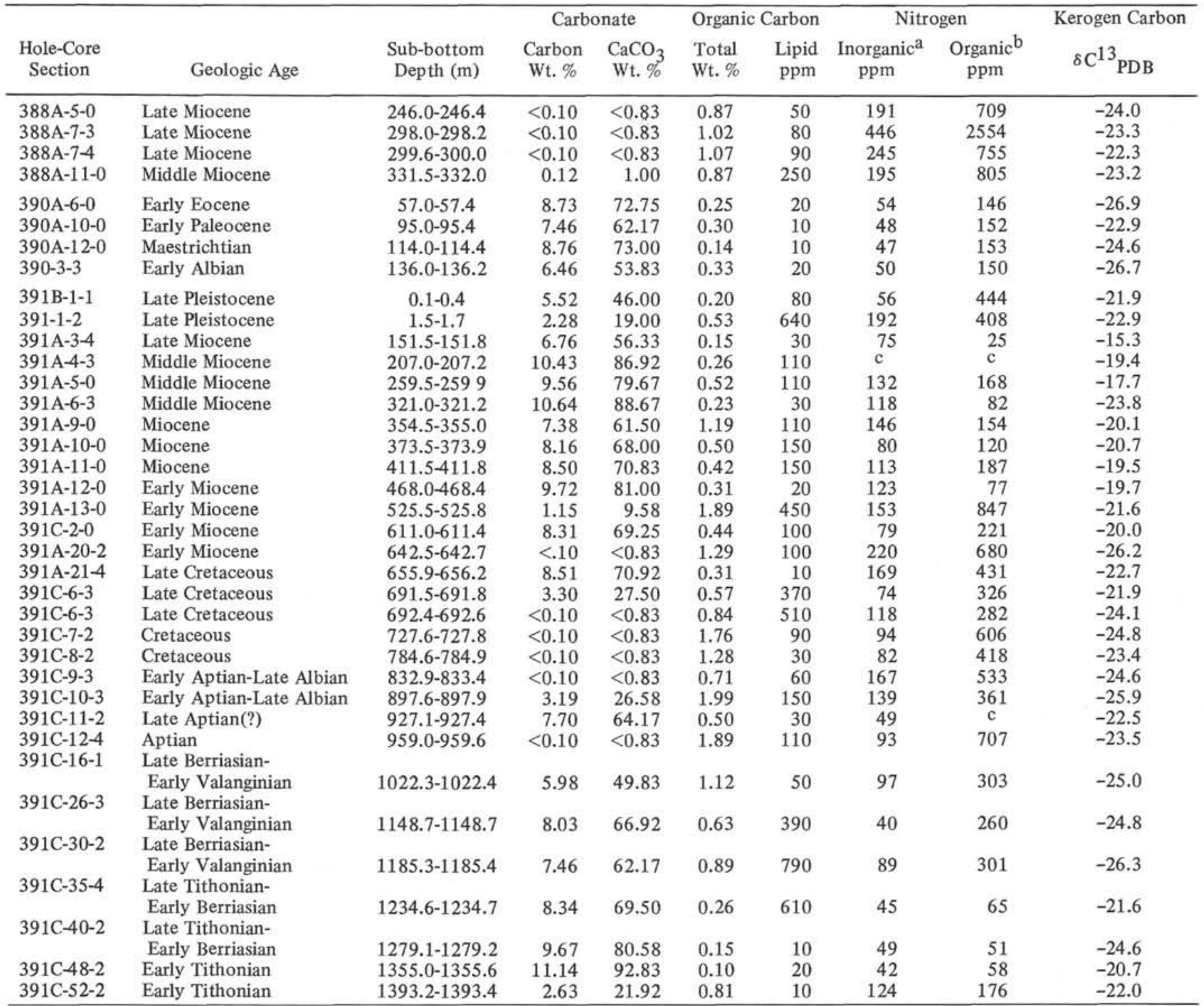

${ }^{\mathrm{a}}$ As determined by the half-Kjeldahl which is representative of ammonium nitrogen only.

${ }^{\mathrm{b}}$ As determined by the full-Kjeldahl minus the half-Kjeldahl.

'Sample insufficient or unsuitable for this determination.

$59(-23.6)$. Only slight variations from these averages were observed throughout the unit (Table 3 ).

The elemental carbon content of the total lipid fraction increases from 74.9 per cent to 77.2 per cent with age or depth. Similarly, the ratio of hydrogen to carbon increases from 1.68 to 1.82 with age and depth, whereas the carbon content and $\mathrm{H} / \mathrm{C}$ ratio of the lipid of Section 391A-13-0 are lower, namely 73.2 and 1.74, respectively, than the previous sections. The sulfur content of all four samples, however, steadily increases with increased depth.

As shown in Figure 7, the $n$-alkane distributions for Sections 391A-4-3, 391A-6-3, 391A-9-0, 391A-13-0, and $391 \mathrm{C}-2-0$ are similar, with major peaks at $n-\mathrm{C}_{27}, n-\mathrm{C}_{29}$, $n-\mathrm{C}_{31}$ with $n-\mathrm{C}_{29}$ predominating. The corresponding $\overline{\mathrm{OEP}}$ values for these samples are high, averaging 2.3
Both the shape of these curves and the OEP values indicate that petroleum genesis has not been extensive. The $n$-alkane distributions for Sections 391A-5-0 and 391A-11-0 are given in Figure 8. Section 391A-5-0 contains a bimodal distribution with a relatively smooth maxima at $n$ - $\mathrm{C}_{25}$ and a typical recent sediment odd-predominance maxima at $n-C_{31}$. The OEP values for Sections 391A-5-0 and 391A-11-0 both suggest more prolific genesis of petroleum.

Unit 1 consists of Pleistocene gray hemipelagic clay. Two samples were obtained at sub-bottom depths of 1.5 and 0.1 meters from Holes 391 and 391B, respectively. Both sediments were poor in organic matter and contained less than 0.6 per cent organic carbon even though chips taken for determination of texture indicated that they were rich in organic matter 
TABLE 3

Chemistry of Core-Sections From DSDP Leg 44 Extract Fractions and Their Elemental and Isotopic Composition

\begin{tabular}{|c|c|c|c|c|c|c|c|c|c|c|c|c|c|c|c|c|}
\hline \multirow{3}{*}{$\begin{array}{l}\text { Hole-Core } \\
\text { Section }\end{array}$} & \multirow{3}{*}{$\begin{array}{c}\text { Lipid/Total } \\
\text { Organic C } \\
\text { (wt. \%) }\end{array}$} & \multirow[b]{3}{*}{$\mathrm{C}$} & \multirow{2}{*}{\multicolumn{7}{|c|}{$\begin{array}{l}\text { Total Lipid } \\
\text { Elemental Composition }\end{array}$}} & \multirow[b]{3}{*}{$8 \mathrm{C}^{13} \mathrm{PDB}$} & \multirow{3}{*}{$\begin{array}{l}\text { Saturate } \\
\text { (wt. \%) }\end{array}$} & \multicolumn{4}{|c|}{ Lipid Fractions } & \multirow[b]{3}{*}{$\overline{\mathrm{OEP}}$} \\
\hline & & & & & & & & & & & & & matic & & haltic & \\
\hline & & & $\mathrm{H}$ & $\mathrm{N}$ & $\mathrm{S}$ & $\mathrm{O}^{\mathrm{b}}$ & $\mathrm{H} / \mathrm{C}$ & $\mathrm{N} / \mathrm{C}$ & $\mathrm{S} / \mathrm{C}$ & & & (wt. \%) & $\left(\delta \mathrm{C}^{13} \mathrm{PDB}\right)$ & (wt. \%) & $\left({ }^{\delta C^{13}} \mathrm{PDB}\right)$ & \\
\hline $388 \mathrm{~A}-5-0$ & 0.59 & & & & & & & & & -26.0 & 22.4 & 29.2 & -26.0 & 48.4 & -25.6 & 2.06 \\
\hline $399 A-7-3$ & 0.81 & & & & & & & & & -26.3 & 18.0 & 20.5 & -26.2 & 61.6 & -26.1 & 2.18 \\
\hline $388 A-7-4$ & 0.80 & & & & & & & & & -25.1 & 16.1 & 19.3 & -25.4 & 64.5 & -24.5 & 1.69 \\
\hline $388 \mathrm{~A}-11-0$ & 2.87 & & & & & & & & & -26.4 & 45.8 & 28.3 & -25.9 & 25.9 & -25.5 & 2.17 \\
\hline $390 A-6-0$ & 0.78 & & & & & & & & & -26.8 & & & & & & \\
\hline $390 A-10-0$ & 0.34 & & & & & & & & & -26.9 & & & & & & \\
\hline $390 \mathrm{~A}-12-0$ & 1.00 & & & & & & & & & -26.4 & & & & & & \\
\hline $390-3-3$ & 0.66 & & & & & & & & & -27.8 & & & & & & \\
\hline $391 B-1-1$ & 4.11 & & & & & & & & & -26.5 & & & & & & \\
\hline $391-1-2$ & 12.04 & 52.1 & 7.7 & 1.9 & & & 1.7 & 0.03 & & -27.6 & 10.8 & 22.1 & -26.4 & 67.1 & -26.6 & 2.14 \\
\hline $391 \mathrm{~A}-3-4$ & 2.01 & & & & & & & & & -23.4 & & & & & & \\
\hline $391 \mathrm{~A}-4-3$ & 4.08 & & & & & & & & & -25.9 & 14.0 & 21.9 & -23.3 & 64.1 & -26.2 & 2.02 \\
\hline $391 \mathrm{~A}-5-0$ & 2.07 & & & & & & & & & -22.8 & 14.3 & 23.9 & -25.2 & 61.8 & -22.6 & 1.31 \\
\hline $391 A-6-3$ & 1.35 & & & & & & & & & -24.3 & 21.0 & 32.6 & -26.0 & 46.4 & -23.1 & 3.10 \\
\hline $391 \mathrm{~A}-9-0$ & 0.89 & 74.9 & 10.5 & 0.3 & 1.01 & 13.3 & 1.68 & 0.003 & 0.005 & -24.9 & 5.4 & 63.8 & -26.2 & 30.8 & -23.3 & 1.97 \\
\hline $391 \mathrm{~A}-10-0$ & 3.07 & 75.7 & 11.2 & 0.1 & 1.9 & 11.2 & 1.77 & 0.001 & 0.009 & -23.4 & 10.9 & 29.5 & -24.7 & 59.5 & -22.4 & \\
\hline $391 \mathrm{~A}-11-0$ & 3.54 & 77.2 & 11.7 & 0.4 & 3.2 & 7.5 & 1.82 & 0.005 & 0.016 & -23.9 & 10.0 & 25.7 & -25.7 & 64.3 & -23.6 & 1.02 \\
\hline $391 \mathrm{~A}-12-0$ & 0.57 & & & & & & & & & -23.1 & & & & & & \\
\hline $391 \mathrm{~A}-13-0$ & 2.37 & 73.2 & 10.6 & 0.9 & 5.8 & 9.5 & 1.74 & 0.011 & .0 .030 & -23.7 & 3.6 & 28.9 & -25.3 & 67.5 & -23.7 & 2.15 \\
\hline $391 C-2-0$ & 2.27 & 76.0 & 10.9 & 0.3 & 2.1 & 10.7 & 1.72 & 0.003 & 0.010 & -25.6 & 11.4 & 61.2 & -26.3 & 27.4 & -24.5 & 1.79 \\
\hline $391 \mathrm{~A}-20-2$ & 0.75 & & & & & & & & & -24.8 & & & & & & \\
\hline $391 \mathrm{~A}-21-4$ & 0.36 & & & & & & & & & -27.2 & & & & & & \\
\hline $391 C-6-3$ & 6.58 & 73.5 & 10.0 & 0.2 & 0.1 & 16.2 & 1.63 & 0.002 & $<0.001$ & -27.1 & 3.4 & 88.6 & -26.5 & 8.0 & -27.7 & 1.48 \\
\hline $391 C-6-3$ & 6.10 & & & & & & & & & -26.8 & 4.1 & 85.6 & -26.7 & 10.3 & -28.0 & 1.69 \\
\hline $391 C-7-2$ & 0.50 & & & & & & & & & -28.5 & & & & & & \\
\hline $391 C-8-2$ & 0.27 & & & & & & & & & -28.7 & & & & & & \\
\hline $391 C-9-3$ & 0.86 & & & & & & & & & -28.9 & & & & & & \\
\hline $391 C-10-3$ & 0.76 & 84.5 & 11.6 & 0.3 & & & 1.64 & 0.003 & & -29.9 & 32.8 & 30.0 & -299 & 37.2 & -29.5 & 2.20 \\
\hline $391 C-11-2$ & 0.64 & & & & & & & & & -28.4 & & & & & & \\
\hline $391 C-12-4$ & 0.57 & & & & & & & & & -28.1 & 11.0 & 51.4 & -27.7 & 37.6 & -28.7 & 1.87 \\
\hline $391 C-16-1$ & 0.41 & & & & & & & & & -29.1 & & & & & & \\
\hline $391 \mathrm{C}-26-3$ & 6.23 & & & & & & & & & -27.1 & 4.1 & 83.4 & -26.8 & 12.5 & -28.4 & 1.50 \\
\hline $391 C-30-2$ & 8.85 & 74.4 & 10.2 & 0.2 & 0.1 & 15.1 & 1.65 & 0.003 & $<0.001$ & -27.0 & 1.8 & 87.8 & -26.7 & 10.4 & -29.2 & 1.55 \\
\hline $391 C-35-4$ & 23.51 & 73.3 & 9.9 & 0.2 & 0.2 & 16.4 & 1.61 & 0.006 & 0.001 & -26.7 & 2.4 & 82.8 & -26.8 & 14.8 & -28.0 & 1.11 \\
\hline $391 C-40-2$ & 0.62 & & & & & & & & & -25.7 & & & & & & \\
\hline $391 C-48-2$ & 2.15 & & & & & & & & & -26.5 & & & & & & \\
\hline $391 C-52-2$ & 0.09 & & & & & & & & & -27.3 & & & & & & \\
\hline
\end{tabular}

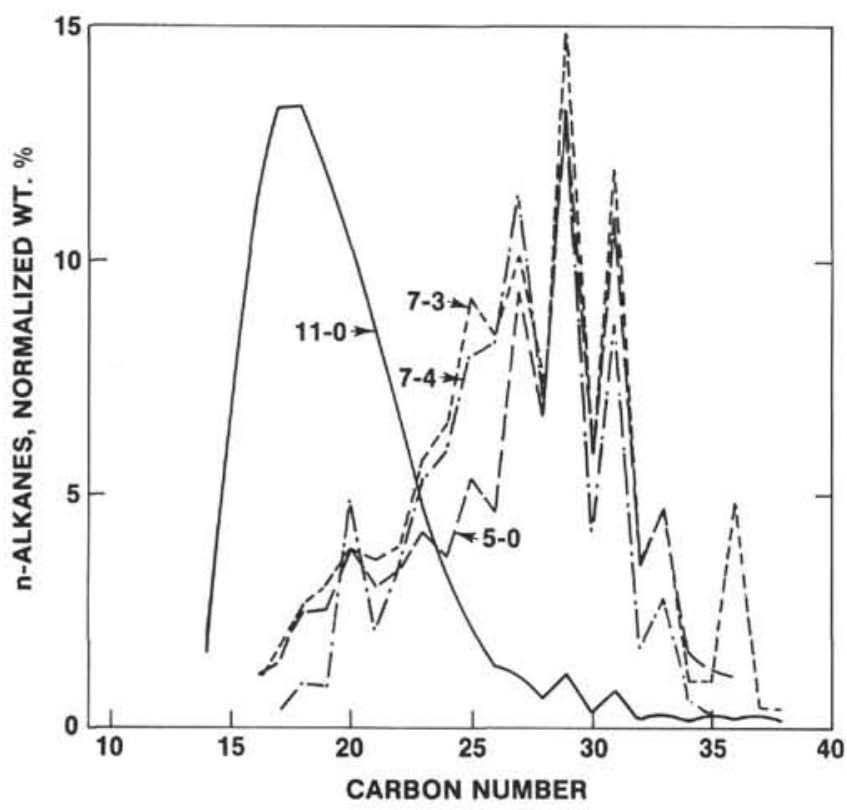

Figure 4. Plots of normalized N-alkane composition versus carbon number for the upper and middle Miocene CoreSections 388A-5-0, 388A-7-3, 388A-7-4, and 388A-11-0.

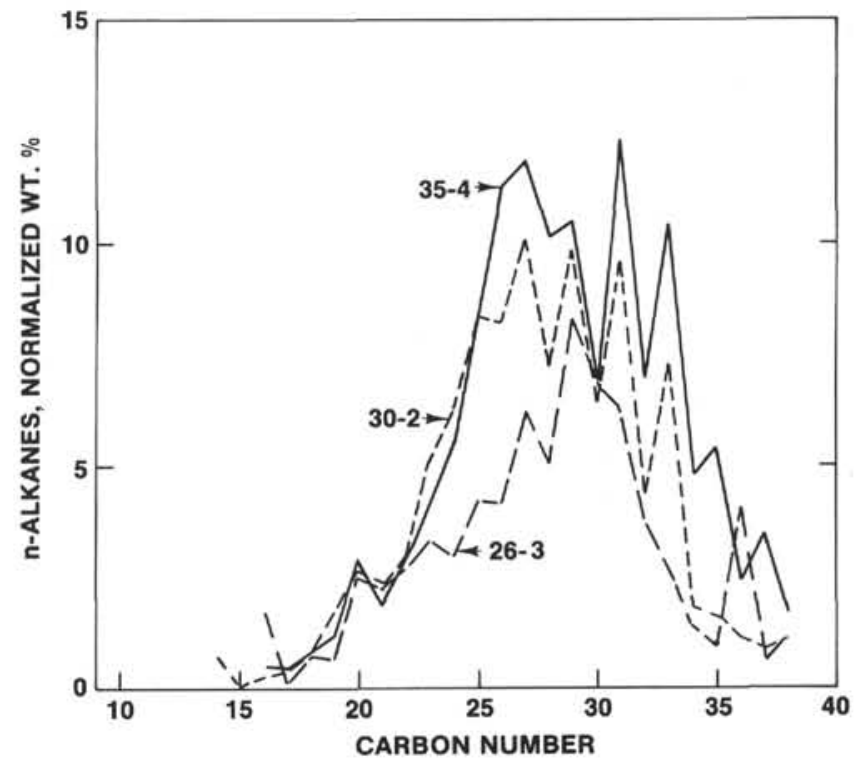

Figure 5. Plots of normalized N-alkane composition versus carbon number for the lower Cretaceous Core-Sections 391C-26-3, 391C-30-2, and 391C-35-4. 


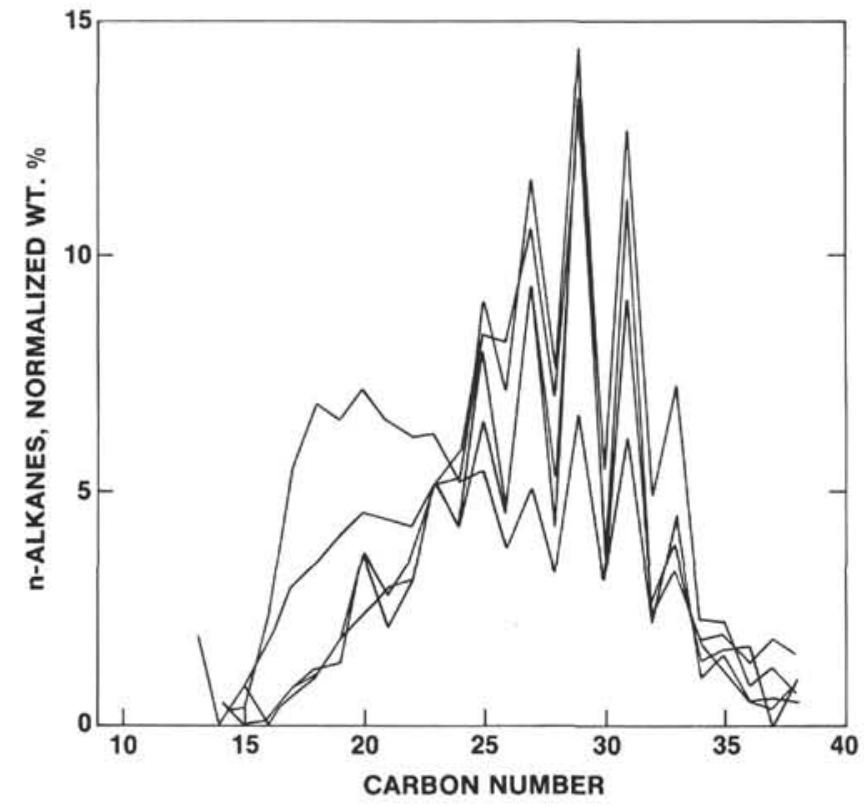

Figure 6. Plots of normalized N-alkane composition versus carbon number for the lower Cretaceous Core-Sections 391C-6-3. 391C-10-3, and 391C-12-4.

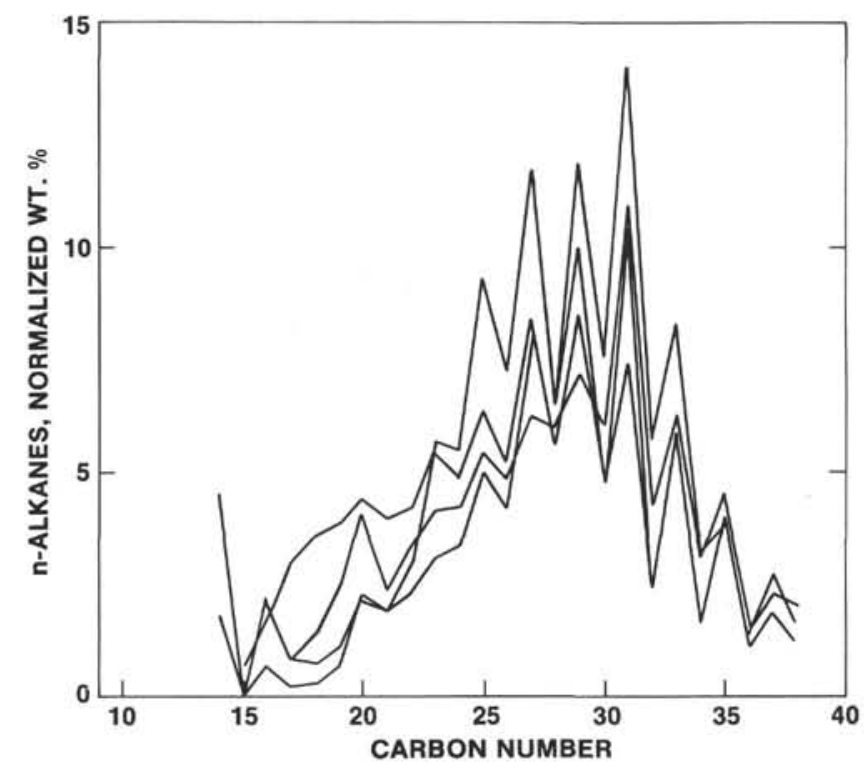

Figure 7. Plots of normalized $\mathrm{N}$-alkane composition versus carbon number for the Miocene Core-Sections 391A-4-3, 391A-6-3, 391A-9-0, 391A-13-0, and 391C-2-0.

(Table 1). The deeper sediment contained 2.28 per cent carbonate carbon (19\% as calcium carbonate), $50 \mathrm{ppm}$ ammonium nitrogen, $150 \mathrm{ppm}$ organic nitrogen, a large fraction of both lipid carbon to total sediment (640 ppm) and to total organic carbon (12 per cent). In contrast, the shallower sediment from this unit contained more carbonate carbon (5.52 per cent) or 46 per cent as calcium carbonate, much less lipid carbon to total sediment $(80 \mathrm{ppm})$ and approximately one-third the amount of lipid. carbon to total organic carbon.

The carbon isotopic compositions of both the lipid and kerogen fractions of the deeper lying sediment were

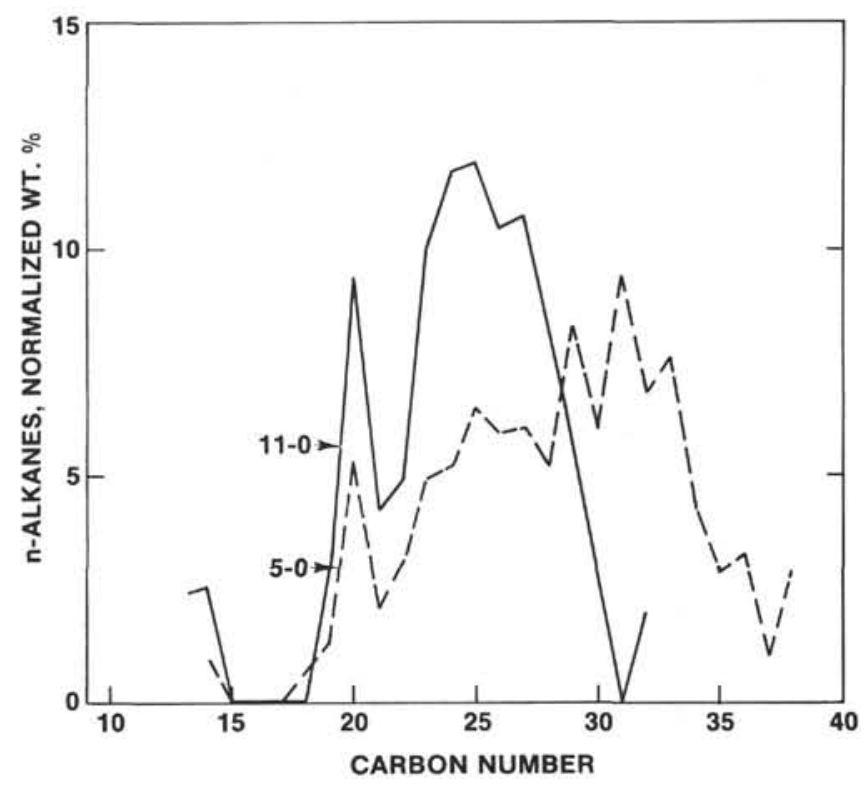

Figure 8. Plots of normalized $\mathrm{N}$-alkane composition versus carbon number for the Miocene Core-Sections 391A-5-0 and 391A-11-0.

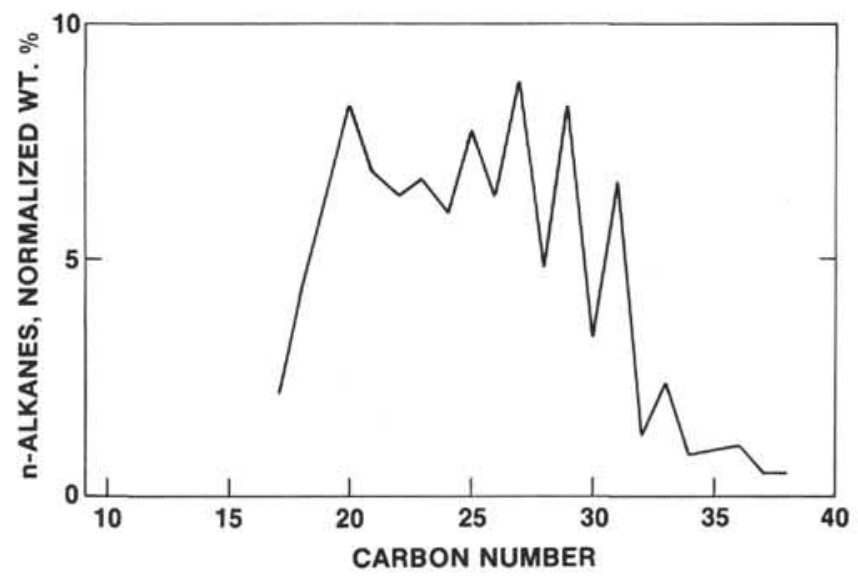

Figure 9. Plots of normalized N-alkane composition versus carbon number for the Pleistocene Core-Section 391-1-2.

lighter than the shallower section. The algebraic differences between the lipid aand kerogen, however, were within experimental error, $-4.65 \pm 0.05$.

Sufficient lipid was present for a total analysis in only the deeper lying sediment. The carbon content of the lipid was 52.1 per cent which is extremely low, and the nitrogen content was 1.9 per cent, which is high. This suggests that the sediment contains a considerable amount of organic matter that has undergone very little diagenetic change.

As shown in Figure 9, the $n$-alkane distribution for Section $391-1-2$ is bimodal with a large odd predominance in the range of $n-\mathrm{C}_{23}$ to $n-\mathrm{C}_{33}$. The curve is relatively smooth in the lower carbon number range.

\section{DISCUSSION}

The 37 core sections from the lower continental rise hills, Blake Nose, and the Blake-Bahama Basin range in 
sub-bottom depth from near the water-sediment interface to approximately 1393 meters, and in age from Pleistocene to Jurassic. Assuming that these sediments were not previously more deeply buried, their temperatures have never been high, and probably did not exceed $45^{\circ}-50^{\circ} \mathrm{C}$. Their ages, however, vary by a factor of nearly two hundred. The inorganic rock matrix varies from almost pure silica-clay minerals to nearly pure calcium carbonate. In general, the organic matter reflects a photosynthetic origin with a marine or open water to nearshore environment. Thus the samples provide the opportunity to observe organic diagenesis at low temperature as a function of time and catalytic activity of the inorganic matrix.

Average odd-even predominance, $\overline{O E P}$, is an indicator of the extent to which $n$-alkanes derived from the living source material are diluted by $n$-alkanes formed by abiogenic chemical processes following burial and cessation of life processes. Stated another way, the $n$-alkanes of biogenic origin, usually with an odd predominance are being diluted with those of abiogenic origin, which have a smooth carbon number distribution, that is, no odd or even predominance. Thus for source material with $n$-alkanes of odd carbon number predominance, the $\overline{\mathrm{OEP}}$ value will decline as organic diagenesis proceeds toward a limiting value of 1.0. Because $n$-alkanes are ubiquitous in liquid petroleum, $\overline{\mathrm{OEP}}$ can be used as a rough indicator of the amount of petroleum formed, particularly when an adjustment is made for the $n$-alkane concentration in the oil on the basis of the composition of the lipid fraction of the rock. Barring migration which usually is not extensive in shallowly buried rocks, the $\overline{\mathrm{OEP}}$ value together with the lipid to total organic carbon ratio becomes an indicator of two interrelated parameters: the capacity of the source organic matter to produce liquid petroleum and the extent to which its genesis has proceeded under the given temperature-time regime (Erdman, 1975a, 1975b, Erdman et al., 1976).

Referring to Table 3, the OEP values are quite low overall and trend to lower values with increasing age. The lower $\overline{\mathrm{OEP}}$ values in conjunction with the relatively high total organic content and the high proportion of saturates and aromatics in the lipid fraction of the older samples lead us to conclude that liquid petroleum genesis is well advanced in some of the older sediment samples. Accordingly, we conclude that (1) petroleum genesis in quantity can proceed at temperatures no higher than $45^{\circ}-50^{\circ} \mathrm{C}$ provided there is a long interval of time and the source material is of the composition and is deposited under favorable conditions for high ultimate yield of petroleum, and (2) petroleum genesis takes place in lime sediments as readily as in clastic sediments.

To provide an accumulation with economic potential or one with a dangerous potential for blowout during uncased drilling, there must be sufficient compaction to induce migration and accumulation in adjacent coarsegrained rocks. Such compaction with subsequent migration, however, has not taken place in the sediments penetrated in these holes.

\section{CONCLUSIONS}

The 37 frozen core samples from the lower continental rise hills, the Blake Nose, and the BlakeBahama Basin are exceptionally rich in organic material; 22 contained more than 0.5 per cent organic carbon. The organic matter was derived mainly from marine organisms. The ages of the samples ranged from Pleistocene to Jurassic. The suite of samples, therefore, provided an excellent opportunity to determine the rate of organic diagenesis and particularly the genesis of petroleum at low temperature.

A well-defined trend in organic diagenesis was observed with increasing age. In the older samples, genesis of liquid petroleum is well advanced. This suggests that for the formation of significant petroleum accumulations, deep burial is required more to provide the compaction necessary for migration rather than the elevated temperature for genesis.

\section{ACKNOWLEDGMENT}

The authors thank J.B. Foresman of Phillips Petroleum Company for his review and suggestions concerning the manuscript and C.P. Elston for her help in obtaining and assembling data presented herein.

\section{REFERENCES}

Erdman, J.G., 1975a. Geochemical formation of oil. In Fischer, A.G. and Judson, S. (Eds.), Petroleum and global tectonics: Princeton, New Jersey (Princeton University Press), p. 225-248.

1975b. Relations controlling oil and gas generation in sedimentary basins: Ninth World Petrol. Congr., Proc., v. 2 , p. $139-148$.

Erdman, J.G., Schorno, K.S., Shveima, J.S., and Juckett, D.A., 1976. Quantitative aspects of the diagenesis of organic matter in sediments: Paper presented, Gordon Research Conference on Organic Geochemistry, Plymouth, New Hampshire, 16-20 August 1976.

Scalan, R.S. and Smith, J.E., 1970. An improved measure of the odd-even predominance in the normal alkanes of sediments, extracts, and petroleum: Geochim. Cosmochim. Acta, v. 34, p. 611-620. 